\title{
Vibration Attenuation Investigations on a Distributed Phononic Crystals Beam for Rubber Concrete Structures
}

\author{
Chao Li $\mathbb{D}^{1},{ }^{1}$ Sifeng Zhang $\left(\mathbb{D},{ }^{1,2}\right.$ Liyong Gao, ${ }^{3}$ Wei Huang, ${ }^{3}$ and Zhaoxin Liu ${ }^{3}$ \\ ${ }^{1}$ School of Transportation Engineering, Shandong Jianzhu University, Jinan, Shandong 250101, China \\ ${ }^{2}$ Shandong Provincial Key Laboratory of Road and Traffic Engineering in Colleges and Universities, Jinan, \\ Shandong 250101, China \\ ${ }^{3}$ Construction Management Branch of Shandong Hi-Speed Group Co., Ltd., Jinan, Shandong 250013, China \\ Correspondence should be addressed to Chao Li; lcseu@foxmail.com and Sifeng Zhang; sddxzsf@sdjzu.edu.cn
}

Received 13 March 2021; Accepted 24 May 2021; Published 7 July 2021

Academic Editor: Ji Wang

Copyright (c) 2021 Chao Li et al. This is an open access article distributed under the Creative Commons Attribution License, which permits unrestricted use, distribution, and reproduction in any medium, provided the original work is properly cited.

\begin{abstract}
Locally resonant phononic crystals (LRPCs) beam is characterized by the band gaps; some frequency ranges within which flexural waves cannot propagate freely. So, the LRPCs beam can be used for noise or vibration isolation. In this paper, a LRPCs beam with distributed oscillators is proposed, and the general formula of band gaps and transmission spectrum are derived by the transfer matrix method (TMM) and spectrum element method (SEM). Subsequently, the parameter effects on band gaps are investigated in detail. Finally, a rubber concrete beam is designed to demonstrate the application of distributed LRPCs beam in civil engineering. Results reveal that the distributed LRPCs beam has multifrequency band gaps and the number of the band gaps is equal to that of the oscillators. Compared with others, the distributed LRPCs beam can reduce the stress concentration when subjected to vibration. The oscillator interval has no effect on the band gaps, which makes it more convenient to design structures. Individual changes of oscillator mass or stiffness affect the band gap location and width. When the resonance frequency of oscillator is fixed, the starting frequency of the band gap remains constant, and increasing oscillator mass of high-frequency band gap widens the high-frequency band gap, while increasing oscillator mass of low-frequency gap widens both high-frequency and low-frequency band gaps. External loads, such as the common uniform spring force provided by foundation in civil engineering, are conducive to the band gap, and when the spring force increases, all the band gaps are widened. Taken together, a configuration of LRPCs rubber concrete beam is designed, and it shows good isolation on the vibration induced by the railway. By the presented design flow chart, the research can serve as a reference for vibration isolation of LRPCs beams in civil engineering.
\end{abstract}

\section{Introduction}

The concept of phononic crystals (PCs) was first proposed by Kushwaha et al. [1]. PCs are usually artificial and periodic materials, consisting of two or more kinds of materials with different properties [2-7]. And they are characterized by the band gaps, some frequency ranges within which elastic waves cannot propagate freely, which can be used for vibration isolation $[8,9]$. Much attention has been focused on PCs for their elastic band gaps at present. And this fundamental property leads to various applications of PCs, such as vibration insulators $[10]$, transducers $[2,3,11]$, and acoustic filters $[12,13]$.

Generally, there are two kinds of interpretations for the band gap mechanism of PCs, including Bragg scattering mechanism [14] and locally resonant (LR) mechanism [15]. The Bragg scatting band gaps are caused by multiple scatterings of the periodic inclusions, and they usually exist in high-frequency ranges, whereas the LR band gaps are caused by the locally resonant vibration of the inclusions, and the frequency range is almost two orders of magnitude lower than that of the Bragg scattering ones with similar structural size. That is, with LR mechanism, low-frequency band gaps can be obtained with PCs in a smaller structural size, while the Bragg scattering mechanism needs a larger structural size [16]. Therefore, the LRPCs are of excellent low-frequency vibration/acoustic isolation characteristics. To figure out the band gaps, many methods have been proposed, including transfer-matrix method (TM), multiple-scattering theory 
(MST), finite difference time-domain method (FDTD), lumped-mass method (LM), plane wave expansion method (PWE), improved plane wave expansion method (IPWE) [17], finite element method (FEM), wave finite element method [18], and boundary element method (BE) [19].

In engineering fields, the beams are the widely used structure types, in which the flexural waves lead mostly to the vibration and acoustic noise [20]. For this, introduction of LR mechanism into beams and fabricating LRPCs beams can contribute to low-frequency vibration isolation. In this respect, Wang et al. [21] firstly introduced the theory of local resonance into beam and calculated and verified the vibration isolation of LRPCs beam. Lately, Yu et al. [22, 23] and Xiao [24] have promoted the previous research. The characteristics of LRPCs beam, vibration isolation in lowfrequency ranges, make it popular and widely applied in engineering fields, such as mechanical engineering $[25,26]$ and civil engineering $[27,28]$. In addition, vibration is mostly presented in multifrequency way in practical engineering. For this, some scholars have proposed multidegree of freedom local resonance beam structures, namely, the LRPCs beam attached with multiple local resonators. And these LRPCs beams are always composed of the local resonators attached in series [29,30] or in parallel [25, 31-33], so as to obtain the multifrequency band gaps. Nevertheless, both the series type and parallel type might induce mass concentration of local resonators at connection point of the beam, which may adversely affect the structure. The concept of local resonator is essentially the same as that of dynamic vibration absorbers (DVAs) in mechanical engineering [34]. Therefore, according to the suspension type of local resonators, the multidegree of freedom local resonance beam can be divided into combined-parallel type and discrete-distributed type as well $[24,31,34]$. The local resonators of discrete-distributed type are attached to the beam at intervals, which can effectively relieve the mass concentration of the beam. Moreover, the beams are usually subjected to external loads in practice. Like civil engineering, existence of beam resting on foundation is often encountered [35], in which the foundation provides a uniform spring force for the beam. Some researchers have devoted a lot to this subject. Yu et al. [36] analyzed the effects of Winkler foundations on the gap properties of LRPCs Euler beam. Zhang et al. [37] proposed a modified transfer matrix method to investigate the effects of Winkler foundation on the band gaps of LRPCs beams. However, these studies are mainly concerned with the LRPCs beams with single resonator. As reviewed, previous researches have analyzed the band gap characteristics of LRPCs beams with distributed resonators. However, the analysis on the band gaps is not comprehensive. For example, the beams are mostly homogeneous while neglecting the periodicity of the beams, and there is no contrastive analysis on the stress of the beam during vibration isolation. Besides, the effects of foundation spring force on the band gaps of LRPCs beams with multiple local resonators are vacancy.

Meanwhile, various kinds of engineering materials have been developed to attenuate nuisance vibration of structures. In civil engineering, tire rubber concrete, a main recycling strategy of the waste tire rubber, has been adopted worldwide for civil engineering recovery [38]. Incorporation of rubber into concrete can significantly improve the dampness ratio of concrete, which makes the rubber concrete have better vibration absorbing capacity, sound absorbing capacity, and seismic resistance [39]. Thus, it can be used for railways sleepers, seismic prone structures, concrete beams and columns, etc. Specially, the common rubber beams are efficient under service loads, as well as in extreme loading conditions [40-42]. It is observed that the rubber beams can increase the primary natural vibration frequency, thereby improving the earthquake resistance [43]. Furthermore, introduction of local resonance theory into rubber beam can form the typical three-component LRPCs materials [15], hard-soft-hard (concrete matrix-rubber coating layer-concrete core). In this case, the LRPCs rubber concrete has the potential to attenuate the low-frequency vibration in civil engineering.

In present study, a LRPCs beam model with multioscillators is proposed to conduct a comprehensive investigation on the band gap properties of the LRPCs beams. Considering the periodicity of the beam, the general formula of band structure and transmission spectrum for LRPCs beam with distributed multioscillators are derived by TMM and SEM, respectively. The derivation is verified by finite element method (FEM). Then, the effects of beam and oscillators on the band gap are investigated in detail. Considering the application in civil engineering, the external loads provided by foundation are included in the model, and the effects of foundation spring force on the band gaps are analyzed as well. Based on this, a LRPCs rubber concrete beam is proposed to demonstrate the application of distributed LRPCs beam with rubber concrete. And the vibration isolation effect of the LRPCs rubber concrete beam is demonstrated. The results can provide reference for the band gap design of LRPCs beam in civil engineering.

\section{Theoretical Model and Mechanism}

2.1. Band Structure Based on TMM. The theoretical model of LRPCs beam with distributed multioscillators $(m \geq 2)$ has the form shown in Figure 1. The model is constructed with multitype oscillators (different mass and spring stiffness) alternately and periodically attached to the multicomponent $(m \geq 2)$ Euler beam at different intervals, $a_{1}, a_{2}$, and $a_{\mathrm{m}}$. The infinite model is composed of the period multioscillators element, also known as primitive cell. The spacing of adjacent primitive cell is called lattice constant, which is expressed as $a$.

To clearly demonstrate the band gap calculation of the multioscillator configuration, a special case $(m=3)$ is taken, and the corresponding primitive cell is shown in Figure 2. As depicted in Figure 2, three types of oscillators (masses $m_{1}$, $m_{2}$, and $m_{3}$, and springs $k_{1}, k_{2}$, and $k_{3}$ ) are alternately attached to a three-component Euler beam. The intervals between adjacent oscillators are $a_{1}, a_{2}$, and $a_{3}$, respectively. In this paper, the transfer matrix method (TMM) is used to calculate the complex band structure. The three-oscillator primitive cell can be regarded as a supercell composed of 


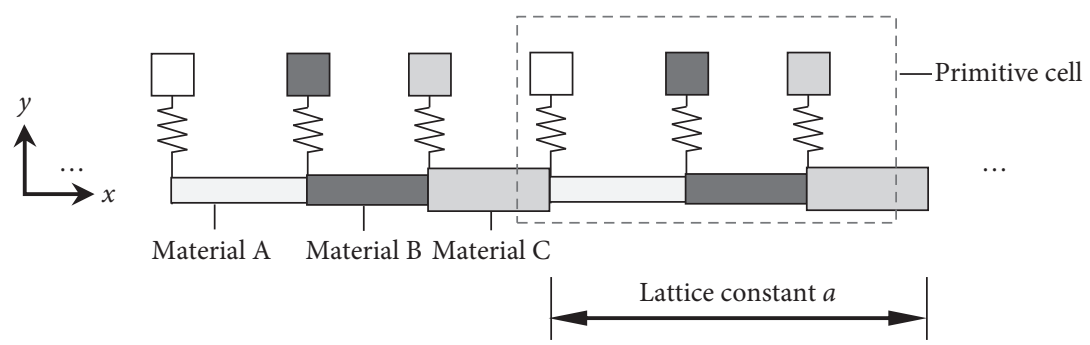

FIgURE 1: Theoretical model of LRPCs beam with distributed multioscillators $(m \geq 2)$.

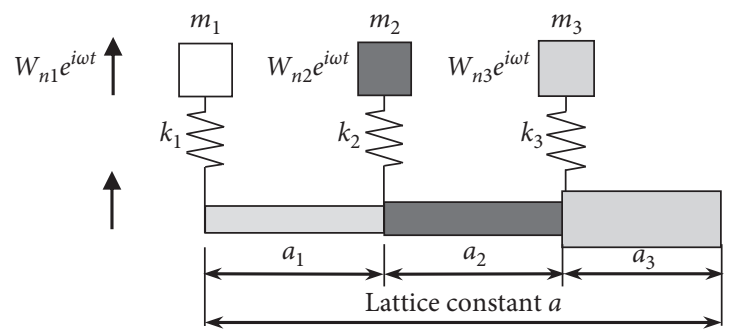

FIgURE 2: The primitive cell of LRPCs beam and the force equilibrium $(m=3)$.

three single-oscillators. Therefore, the complex band structure of LRPCs beam can be solved by the introduction of continuity condition at the interface of the supercell and the periodic boundary condition at the boundary of the supercell. The governing equation for free flexural vibration in Euler-Bernoulli beam can be expressed as

$$
E(x) I(x) \frac{\partial^{4} y(x, t)}{\partial x^{4}}+\rho(x) A(x) \frac{\partial^{2} y(x, t)}{\partial t^{2}}=0,
$$

where $E I$ is the flexural rigidity of the beam; $\rho, A, b$ are density, cross-sectional area, and width of the beam, respectively. These parameters are all the functions of position $x$

For simple harmonic wave, the harmonic solution has the form $y(x, t)=Y(x) e^{-i \omega t}$, where $Y(x)$ is amplitude, and it can be expressed as

$$
\begin{aligned}
Y_{n i}\left(x_{n i}\right)= & A_{n i} \cos \left(\kappa_{i} x_{n i}\right)+B_{n i} \sin \left(\kappa_{i} x_{n i}\right)+C_{n i} \cosh \left(\kappa_{i} x_{n i}\right) \\
& +D_{n} \sinh \left(\kappa_{i} x_{n i}\right), \quad i=1,2, \ldots, m,
\end{aligned}
$$

where $\kappa_{i}=\left[\rho_{i} A_{i} \omega^{2} /\left(E_{i} I_{i}\right)\right]^{1 / 4}, 0 \leq x_{n i} \leq a_{i}, i=1,2, \ldots, m$.

For oscillator at $x=x_{n i}$, the linear displacement of the oscillator can be expressed as

$$
w_{n i}=W_{n i} e^{i \omega t}, \quad i=1,2, \ldots, m
$$

where $W$ is amplitude in vertical direction.

Then, the oscillators satisfy the dynamic equilibrium equation

$$
\left[y_{n}\left(x_{n i}\right)-w_{n i}\right] \cdot k_{i}=m_{i} \ddot{w}_{n i}=-\omega^{2} m_{i} W_{n i}, \quad i=1,2, \ldots, m,
$$

where $k_{i}$ is stiffness of the spring at $x_{n i} ; m_{i}$ is mass of the oscillator at $x=x_{n i}$; $\omega$ is circular frequency.

Equation (4) can be transformed into

$$
W_{n i}=\frac{k_{i}}{k_{i}-\omega^{2} m_{i}} Y\left(x_{n i}\right), \quad x_{n i}=0, i=1,2, \ldots, m .
$$

For periodic beam, the displacement $Y$, rotation angle $Y^{\prime}$, bending moment $E I Y^{\prime \prime}$, and shear force $E I Y^{\prime \prime \prime}$ are the continuous physical quantity at interfaces of $x=n a+a_{1}$, $x=n a+a_{2}$ and $x=n a$.

The transmission matrix of the LRPCs beam with distributed $m$-oscillators can be obtained as follows:

$$
\boldsymbol{\Psi}_{n m}=\mathbf{K}_{m}^{-1} \mathbf{H}_{m-1} \mathbf{K}_{m-1}^{-1} \ldots \mathbf{H}_{2} \mathbf{K}_{2}^{-1} \mathbf{H}_{1} \mathbf{K}_{1}^{-1} \mathbf{H}_{m} \boldsymbol{\Psi}_{(n-1) m}=\mathbf{L} \boldsymbol{\Psi}_{(n-1) m},
$$

where $\mathbf{L}$ is the transfer matrix, which describes the propagation of flexural wave in $x$ direction, $\Psi_{n i}=\left[\begin{array}{llll}A_{n i} & B_{n i} & C_{n i} & D_{n i}\end{array}\right]^{T}, i=1,2, \ldots, m$, 


$$
\begin{aligned}
\mathbf{K}_{i} & =\left(\begin{array}{cccc}
1 & 0 & 1 & 0 \\
0 & \kappa_{i} & 0 & \kappa_{i} \\
-E_{i} I_{i} \kappa_{i}^{2} & 0 & E_{i} I_{i} \kappa_{i}^{2} & 0 \\
F_{n i} & -E_{i} I_{i} \kappa_{i}^{3} & F_{n i} & E_{i} I_{i} \kappa_{i}^{3}
\end{array}\right), \\
\mathbf{F}_{n i} & =-\frac{\cosh \left(\kappa_{i} a_{i}\right)}{\left(\begin{array}{c}
\left.1-\left(\omega^{2} m_{i} / k_{i}\right)\right) \\
\omega^{2}
\end{array}\right.} \\
\mathrm{H}_{i} & =\left(\begin{array}{cccc}
\cos \left(\kappa_{i} a_{i}\right) & \sinh \left(\kappa_{i} a_{i}\right) \\
-\kappa_{i} \sin \left(\kappa_{i} a_{i}\right) & \kappa_{i} \cos \left(\kappa_{i} a_{i}\right) & \kappa_{i} \sinh \left(\kappa_{i} a_{i}\right) & \kappa_{i} \cosh \left(\kappa_{i} a_{i}\right) \\
-E_{i} I_{i} \kappa_{i}^{2} \cos \left(\kappa_{i} a_{i}\right) & -E_{i} I_{i} \kappa_{i}^{2} \sin \left(\kappa_{i} a_{i}\right) & E_{i} I_{i} \kappa_{i}^{2} \cosh \left(\kappa_{i} a_{i}\right) & E_{i} I_{i} \kappa_{i}^{2} \sinh \left(\kappa_{i} a_{i}\right) \\
E_{i} I_{i} \kappa_{i}^{3} \sin \left(\kappa_{i} a_{i}\right) & -E_{i} I_{i} \kappa_{i}^{3} \cos \left(\kappa_{i} a_{i}\right) & E_{i} I_{i} \kappa_{i}^{3} \sinh \left(\kappa_{i} a_{i}\right) & E_{i} I_{i} \kappa_{i}^{3} \cosh \left(\kappa_{i} a_{i}\right)
\end{array}\right) .
\end{aligned}
$$

Furthermore, with the introduction of the periodic boundary conditions, namely, Bloch-Floquet theorem [36], the above problem can be transformed into the standard eigenvalue problem as follows:

$$
\left(\mathbf{L}-e^{i q a} \mathbf{I}\right) \boldsymbol{\Psi}_{(n-1) m}=0 .
$$

By the solution of the eigenvalues of the matrix equation (8), the standard eigenvalue equation of the LRPCs beam with $m$ oscillators can be obtained as follows:

$$
\left|\mathbf{L}-e^{i q a} \mathbf{I}\right|=0 .
$$

Equation (9) is the general formula of complex band structure for distributed LRPCs beam considering periodicity of beam. From equation (9), the frequency-wave vector structure, namely, complex band structure, can be obtained by different values of circular frequency $\omega$. According to Refs. [32, 44], the frequency ranges corresponding to imaginary wave vector are the band gaps, and the ones corresponding to real wave vector are the passing bands.

2.2. Transmission Spectrum Based on SEM. In practice, the LRPCs beam structure is of finite length, and thus, the vibration transmission spectrum is calculated based on SEM in this paper. The finite length LRPCs beam is shown in Figure 3. Similar to FEM, $n$ resonators are distributed on the finite length beam, dividing the beam into $n$ beam elements and $n+1$ nodes. Each beam is a spectral element. The element number is $1,2, \ldots, j, \ldots, n$, and the node number is 1 , $2,3, \ldots, i, \ldots, n, n+1$.

The dynamic stiffness matrix of spectral element $j$ can be expressed as

$$
\mathbf{D}_{j}=\left[\begin{array}{ll}
\mathbf{D}_{j, 11} & \mathbf{D}_{j, 12} \\
\mathbf{D}_{j, 21} & \mathbf{D}_{j, 22}
\end{array}\right],
$$

where each submatrix can be expressed as

$$
\begin{aligned}
& \mathbf{D}_{j, 11}=\frac{E_{j} I_{j}}{a_{j}^{3}}\left[\begin{array}{cc}
\alpha & \bar{\gamma} a_{j} \\
\bar{\gamma} a_{j} & \beta a_{j}^{2}
\end{array}\right], \\
& \mathbf{D}_{j, 12}=\mathbf{D}_{j, 21}^{T}=\frac{E_{j} I_{j}}{a_{j}^{3}}\left[\begin{array}{cc}
-\bar{\alpha} & \gamma a_{j} \\
-\gamma a_{j} & \bar{\beta} a_{j}^{2}
\end{array}\right], \\
& \mathbf{D}_{j, 22}=\frac{E_{j} I_{j}}{a_{j}^{3}}\left[\begin{array}{cc}
\alpha & -\bar{\gamma} a_{j} \\
-\bar{\gamma} a_{j} & \beta a_{j}^{2}
\end{array}\right] .
\end{aligned}
$$

The elements of the matrix are

$$
\begin{aligned}
& \alpha=\frac{\left[\cos \left(k_{b} a_{j}\right) \sinh \left(k_{b} a_{j}\right)+\sin \left(k_{b} a_{j}\right) \cosh \left(k_{b} a_{j}\right)\right]\left(k_{b} a_{j}\right)^{3}}{\Delta}, \\
& \bar{\alpha}=\frac{\left[\sin \left(k_{b} a_{j}\right)+\sinh \left(k_{b} a_{j}\right)\right]\left(k_{b} a_{j}\right)^{3}}{\Delta}, \\
& \beta=\frac{\left[-\cos \left(k_{b} a_{j}\right) \sinh \left(k_{b} a_{j}\right)+\sin \left(k_{b} a_{j}\right) \cosh \left(k_{b} a_{j}\right)\right]\left(k_{b} a_{j}\right)}{\Delta},
\end{aligned}
$$$$
\bar{\beta}=\frac{\left[-\sin \left(k_{b} a_{j}\right)+\sinh \left(k_{b} a_{j}\right)\right]\left(k_{b} a_{j}\right)}{\Delta},
$$$$
\gamma=\frac{\left[-\cos \left(k_{b} a_{j}\right)+\cosh \left(k_{b} a_{j}\right)\right]\left(k_{b} a_{j}\right)^{2}}{\Delta},
$$$$
\bar{\gamma}=\frac{\sin \left(k_{b} a_{j}\right) \sinh \left(k_{b} a_{j}\right)\left(k_{b} a_{j}\right)^{2}}{\Delta},
$$$$
\Delta=1-\cos \left(k_{b} a_{j}\right) \cosh \left(k_{b} a_{j}\right)
$$

$k_{b}=\left(\frac{\rho_{j} A_{j} \omega^{2}}{E_{j} I_{j}}\right)^{1 / 4}$. 


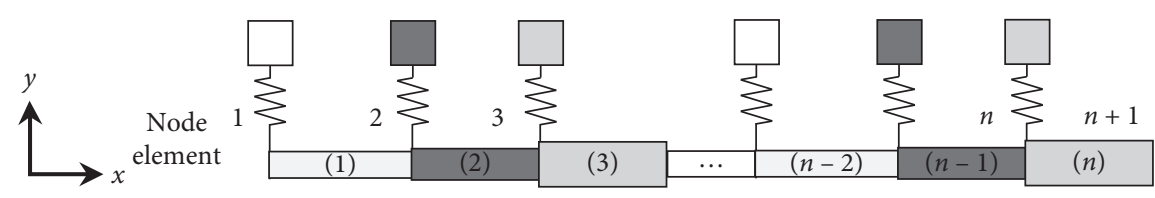

FIgURE 3: Setting for the element and node of finite LRPCs beam.

The additional dynamic stiffness at the attachment point of each resonator is

$$
D_{w i}=\frac{F_{i, 0}}{W_{i, 0}}=\frac{-\omega^{2} m_{w i, 1} k_{w i}}{k_{w i}-\omega^{2} m_{w i, 1}}-m_{w i, 0} \omega^{2}, \quad i=1,2, \ldots, m,
$$

The action of resonators on the beam can be expressed in additional dynamic stiffness matrix form:

$$
D_{i, 0}=\left[\begin{array}{cc}
D_{w i} & 0 \\
0 & 0
\end{array}\right], \quad i=1,2, \ldots, m
$$

$$
\mathbf{D}_{\mathbf{b}}=\left[\begin{array}{ccccccc}
\mathbf{D}_{1,11} & \mathbf{D}_{1,12} & 0 & 0 & \ldots & 0 & 0 \\
\mathbf{D}_{1,21} & \mathbf{D}_{1,22}+\mathbf{D}_{2,11}+\mathbf{D}_{1,0} & \mathbf{D}_{2,12} & 0 & \vdots & \vdots & 0 \\
0 & \mathbf{D}_{2,21} & \mathbf{D}_{2,22}+\mathbf{D}_{3,11}+\mathbf{D}_{2,0} & \mathbf{D}_{3,12} & \ldots & \ldots & 0 \\
0 & 0 & \vdots & \ddots & \vdots & 0 & \mathbf{D}_{n, 12} \\
\vdots & \ldots & \vdots & \mathbf{D}_{n-1,21} & \mathbf{D}_{n-1,22}+\mathbf{D}_{n, 11}+\mathbf{D}_{m-1,0} & \mathbf{D}_{n, 22}+\mathbf{D}_{n+1,11}+\mathbf{D}_{m, 0} & \mathbf{D}_{n+1,12} \\
0 & 0 & \vdots & 0 & \mathbf{D}_{n, 21} & \mathbf{D}_{n+1,21} & \mathbf{D}_{n+1,22}
\end{array}\right] .
$$

By employing equation (15), the dynamic equation of LRPCs beam with multioscillators can be expressed as

$$
\mathbf{D}_{b} \mathbf{u}=\mathbf{f},
$$

where generalized coordinates $\mathbf{u}=\left[\begin{array}{llll}\mathbf{u}_{1} & \mathbf{u}_{2} & \ldots & \mathbf{u}_{n+1}\end{array}\right]^{T}$, $\mathbf{u}_{1}=\left[\begin{array}{ll}w_{i} & \theta_{i}\end{array}\right]^{T}, \mathbf{u}_{n+1}=\left[\begin{array}{ll}w_{o} & \theta_{o}\end{array}\right]^{T}$; external excitation load $\mathbf{f}=\left[\begin{array}{llll}\mathbf{f}_{1} & 0 & \ldots & 0\end{array}\right]^{T}, \mathbf{f}_{1}=\left[\begin{array}{ll}f_{1} & 0\end{array}\right]^{T}$.

Applying load at the left end of LRPCs beam and picking up the displacement vibration response at both ends of LRPCs beam, we can obtain the vibration transmission spectrum of the LRPCs beam as follows:

$$
T=20 \log \left[\frac{w_{o}(\omega)}{w_{i}(\omega)}\right]
$$

where the unit of $T$ is in $\mathrm{dB}$.

Equation (17) is the general form of transmission spectrum for LRPCs beam with distributed multioscillators.

2.3. Verification of the Method. To verify the theoretical derivation, a special calculation model $(m=2)$ is taken. The materials are as follows: the beam, width $b_{1}=b_{2}=0.02 \mathrm{~m}$, height $h_{1}=h_{2}=0.004 \mathrm{~m}$, elasticity modulus

$E_{1}=21.06 \times 10^{10} \mathrm{~Pa}, E_{2}=7.76 \times 10^{10} \mathrm{~Pa}$, density $\rho_{1}=7780 \mathrm{~kg} /$ $\mathrm{m}^{3}, \rho_{2}=2730 \mathrm{~kg} / \mathrm{m}^{3}$. The oscillators are displaced at equal intervals, $a_{1}=a_{2}=0.015 \mathrm{~m}$ : oscillators, $m_{1}=9.28 \times 10^{-3} \mathrm{~kg}$, $k_{1}=3.29 \times 10^{5} \mathrm{~N} / \mathrm{m}, m_{2}=3.71 \times 10^{-2} \mathrm{~kg}, k_{2}=3.29 \times 10^{5} \mathrm{~N} / \mathrm{m}$. According to equation (9), the complex band structure of the LRPCs beam can be obtained. Figure 4 illustrates the imaginary band structure of LRPCs beam with distributed multioscillators $(m=2)$.

As shown in Figure 4, the LRPCs beam has two band gaps, located in the ranges of $469 \sim 813 \mathrm{~Hz}$ and $943 \sim 1475 \mathrm{~Hz}$, respectively.

According to equation (17), the vibration transmission spectrum of LRPCs beam with distributed multioscillators can be calculated to demonstrate its attenuation characteristics. Furthermore, a FEM beam model ( 6 cells) with the same configurations is constructed to verify the theoretical derivation. The simulation model is shown in Figure 5, in which tetrahedral elements are adopted with an element number of 16899 . The material and structural parameters of the FEM model are displayed in Tables 1 and 2 , respectively.

Apply the acceleration excitation at one end of the finite element structure and pick it up at the other end to calculate the transmission spectrum. The transmission spectrum of
Assembling the dynamic stiffness matrix of each spectral element with the additional dynamic stiffness matrix of each oscillator can obtain the dynamic stiffness matrix of each spectral element. By assembling the dynamic stiffness matrix of each spectral element, we can obtain the dynamic stiffness matrix of the whole LRPCs beam. The dynamic stiffness matrix of the LRPCs beam can be expressed as 


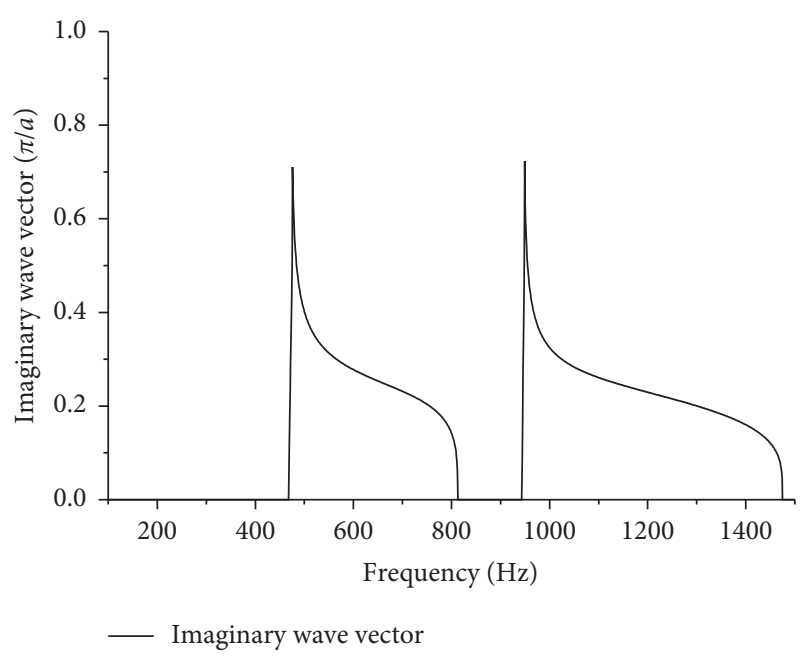

FIgURE 4: Imaginary band structure of LRPCs beam $(m=2)$.

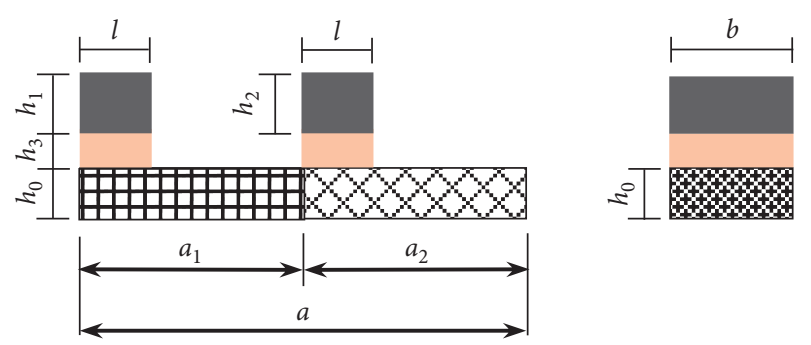

FIgURE 5: Finite element model of LRPCs beam with multioscillators $(m=2)$.

LRPCs beam calculated by theoretical and finite element simulation is shown in Figure 6.

It can be seen from Figure 6 that the theoretical calculation results agree well with those of the finite element simulation, and the transmission coefficient shows obvious attenuation in the ranges of $469 \sim 800 \mathrm{~Hz}$ and $940 \sim 1464 \mathrm{~Hz}$, which are in accordance with the ranges shown in Figure 4. It indicates that there are two frequency attenuation band gaps (ranges in shadows), and the theoretical derivation is verified.

2.4. Vibration Mechanism of the LRPCs Beam. To demonstrate the vibration mechanism of LRPCs beam with multioscillators, taking $m=2$ as an example (the same as Figure 4), the two low-frequency band gaps of the LRPCs beams are all locally resonant ones caused by the local resonance of oscillators. Besides, other cases are also calculated. For example, when $m=3$, there are three low-frequency band gaps, and the rest $(m>3)$ can also be done in the same manner. In conclusion, the number of the locally resonant gaps is equal to that of the local resonators of the distributed LRPCs beam.

Though the locally resonant band gaps are of low frequencies, the local resonance of oscillators may cause vibration stress concentration at attachment point of the beam. In this case, this paper chooses the parameters in reference [31] and calculates the vibration stress of the parallel LRPCs beam and distributed LRPCs beam, respectively. The vibration stress nephogram of the two types of LRPCs beam (excitation frequency $600 \mathrm{~Hz}$ ) is compared in Figure 7.

As depicted in Figure 7, when excitation is located in the attenuation band gap $(600 \mathrm{~Hz})$, the beam suffers vibration stress concentration caused by the resonance of oscillators at the attachment points, especially the points near the excitation. Even so, the fact is that the vibration stress concentration degree of distributed model is greatly decreased compared with that of the parallel model. Therefore, the distributed LRPCs beam can better prevent the beam body from vibration damages. Moreover, calculation shows that the isolation effects of the two models are almost the same. It indicates that the LRPCs beams with distributed multioscillators are of advantage in reducing vibration stress concentration in beam body.

\section{Parameter Study}

3.1. Effects of Interval between Distributed Oscillators on Band Gaps. In order to investigate the influence law of the interval between distributed oscillators on the band gaps of LRPCs beam, we also take the LRPCs beam model with 2 oscillators ( $m=2$, the same as Part 2.3) as an example. For convenience, the periodic beam is degenerated into a homogeneous one (Material 1), and the frequency and oscillator parameters have dealt with dimensionless method to obtain the general law of the effects of interval on the band gaps. The dimensionless mass of the oscillator is defined as the ratio of the oscillator mass to the mass of the corresponding beam segment:

$$
M_{i}=\frac{m_{i}}{\rho A a} .
$$

The dimensionless stiffness of the spring is defined as the ratio of the spring stiffness to the bending stiffness of the corresponding beam segment:

$$
K_{i}=\frac{k_{i}}{E I / a^{3}} .
$$

The dimensionless frequency is defined as the ratio of lattice constant to half wavelength:

$$
\Omega=\frac{a}{\lambda_{b} / 2}=\frac{a}{\pi}\left[\frac{\rho A(2 \pi f)^{2}}{E I}\right]^{1 / 4} .
$$

According to equations (15)-(17), the dimensionless mass, stiffness, and resonance frequency of oscillator 1 and oscillator 2 are calculated as follows: $M_{1}=0.497, M_{2}=1.987$; $K_{1}=0.395, K_{2}=0.395 ; \Omega_{1}=0.301, \Omega_{2}=0.213$. Defining oscillator interval ratio, $n=a_{1} / a$, to analyze the influence law of oscillator interval on the band gap of LRPCs beam, and $n$ ranges from 0 to 1 . The effects of interval on the band gap of LRPCs beam are shown in Figure 8 .

As shown in Figure 8, the oscillator interval shows no effect on either the position or the width of the band gap of the LRPCs beam at fixed beam parameters. It indicates that the oscillator interval has no effect on the band gap 
TABLE 1: Material parameters of FEM beam model.

\begin{tabular}{lccc}
\hline Material & Density $\left(\mathrm{kg} / \mathrm{m}^{3}\right)$ & Young modulus $(\mathrm{Pa})$ & Shear elasticity $(\mathrm{Pa})$ \\
\hline Beam 1 & 7780 & $21.06 e 10$ & $8.10 e 10$ \\
Beam 2 & 2730 & $7.76 e 10$ & $2.87 e 10$ \\
Oscillator 1 & 8950 & $16.46 e 10$ & $7.53 e 10$ \\
Oscillator 2 & 19500 & $8.5 e 10$ & $2.99 e 10$ \\
Silicone rubber & 1300 & $1.0 e 6$ & $3.4 e 5$ \\
\hline
\end{tabular}

TABLE 2: Structural parameters of the FEM beam model.

\begin{tabular}{lccccccc}
\hline$b(\mathrm{~m})$ & $l(\mathrm{~m})$ & $a_{1}(\mathrm{~m})$ & $a_{2}(\mathrm{~m})$ & $h_{0}(\mathrm{~m})$ & $h_{1}(\mathrm{~m})$ & $h_{2}(\mathrm{~m})$ & $h_{3}(\mathrm{~m})$ \\
\hline 0.02 & 0.00833 & 0.015 & 0.015 & 0.004 & 0.00621 & 0.01144 & 0.001 \\
\hline
\end{tabular}

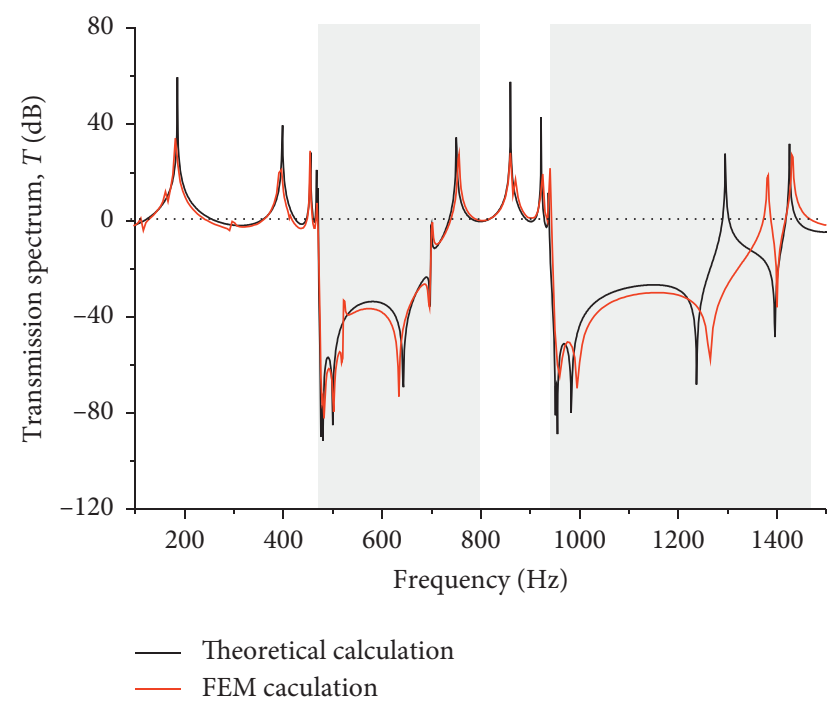

FIGURE 6: Transmittance spectrum of finite LRPCs beam (6 cells).

characteristics of LRPCs beam when the beam is selected. In fact, because of the low-frequency band gaps, the starting frequencies of the band gap are approximately equal to the resonance frequency of the oscillators [24]. When the oscillator interval ratio is 0 or 1 , the LRPCs beam with distributed double-oscillators is transformed into the parallel type $[31,34]$. However, the parallel type may suffer vibration stress concentration at the attachment points. Therefore, transforming the parallel type beam into the distributed one can reduce the stress concentration, while the band gaps are the same.

3.2. Effects of Oscillator Parameters on Band Gaps. Analogously, we can obtain the effects of the oscillator parameters on the band gaps of LRPCs beam. For convenience, we take LRPCs beam model with 2 oscillators $(m=2$, the same as Part 2.3) as an example. Also, we can obtain the general influence law of oscillator parameters after the nondimensional disposal of frequency and oscillator parameters.

3.2.1. Mass of the Oscillator. With the mass of oscillator 2 kept constant and only the dimensionless mass of oscillator 1 varying from 0 to 5.0, the mass effects of oscillator 1 on the band gap of LRPCs beam can be analyzed. The band gap property of LRPCs beam is shown in Figure 9.

As shown in Figure 9, with the mass increase of oscillator 1 , the variation of the LRPCs beam band gap can be divided into three stages: double-band gaps stage, coupled singleband gap stage, and separated-band gaps stage. In the first stage, the mass of oscillator 1 is small, and there are two independent band gaps with the beam, in which the highfrequency band gap is generated by the resonance of oscillator 1 , and the low-frequency band gap is generated by the resonance of oscillator 2 . When the mass of oscillator 1 increases, the resonance frequency of oscillator 1 decreases, and the resonance intensity increases. As a result, the highfrequency band gap gradually moves towards low frequency, and the band gap gradually widens, while the low-frequency band gap is gradually compressed and narrowed, which is similar to the variation rule of the band gap in parallel beam with two oscillators [31]. In the second stage, the resonance frequency of oscillator 1 is equal to that of oscillator 2 , and the two band gaps overlap and couple together into a wide single-band gap. It indicates that the two locally resonant band gaps can couple together precisely. In the third stage, the further mass increase of oscillator 1 makes the single band gap split into two band gaps, in which the low-frequency one is generated by the resonance of oscillator 1, and the high-frequency one is generated by the resonance of oscillator 2. As the mass of oscillator 1 increases, its resonance frequency decreases, which makes the low-frequency band gap move further toward lower frequency. Meanwhile, the low-frequency band gap gradually widens because it moves away from the high-frequency band gap, and its resonance intensity increases. During this process, the position and width of the high-frequency band gap remain nearly stable.

Similarly, with only the mass of oscillator 2 varying, the mass influence of oscillator 2 on the band gaps of LRPCs beam can be obtained. The diagram is shown in Figure 10. As can be seen from Figure 10, the mass of oscillator 2 shows similar effects on the band gap of LRPCs beam to that of oscillator 1 .

The above variation indicates that increasing the mass of oscillator in high-frequency band gap can lower the position of the high-frequency band gap and increase its width, but the low-frequency band gap will be compressed and thus 


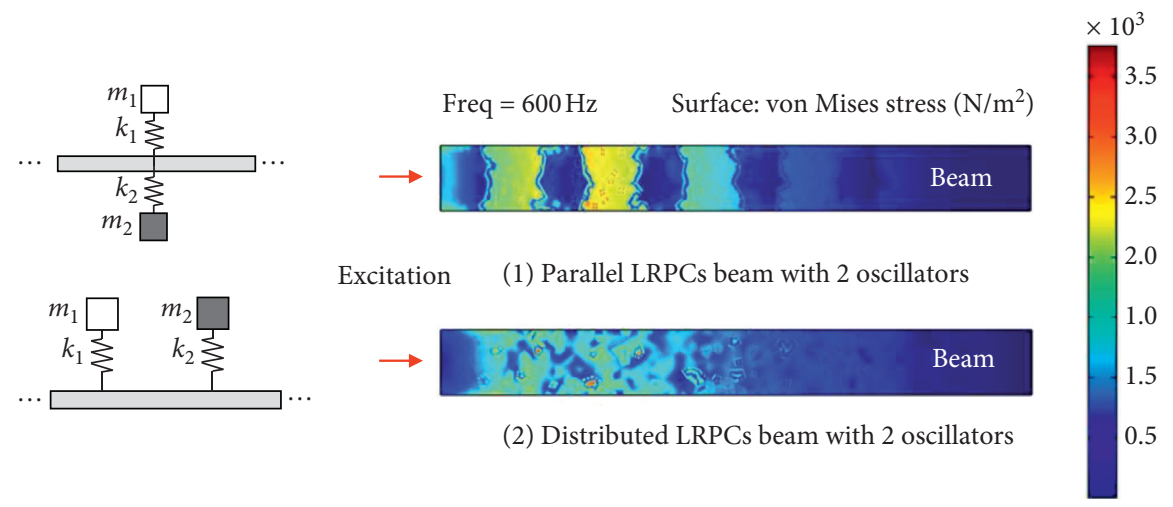

Figure 7: The vibration stress nephogram of different types of LRPCs beam.

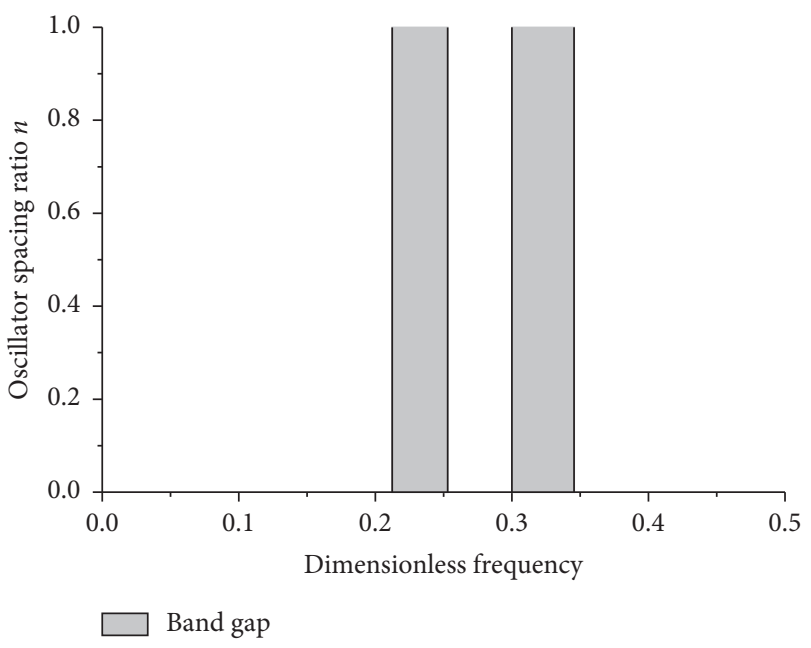

FIGURE 8: Effects of oscillator interval on band gap of LRPCs beam.

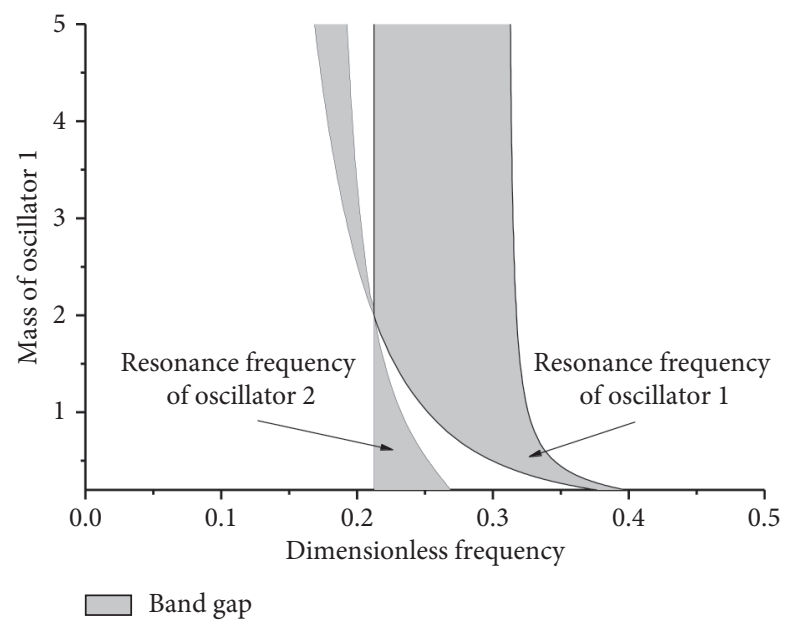

FIGURE 9: Mass effects of oscillator 1 on band gap of the LRPCs beam.

narrows. On the other hand, increasing the mass of oscillator in low-frequency band gap can lower the position of lowfrequency band gap and increase its width, while the highfrequency band gap remains nearly unaffected.

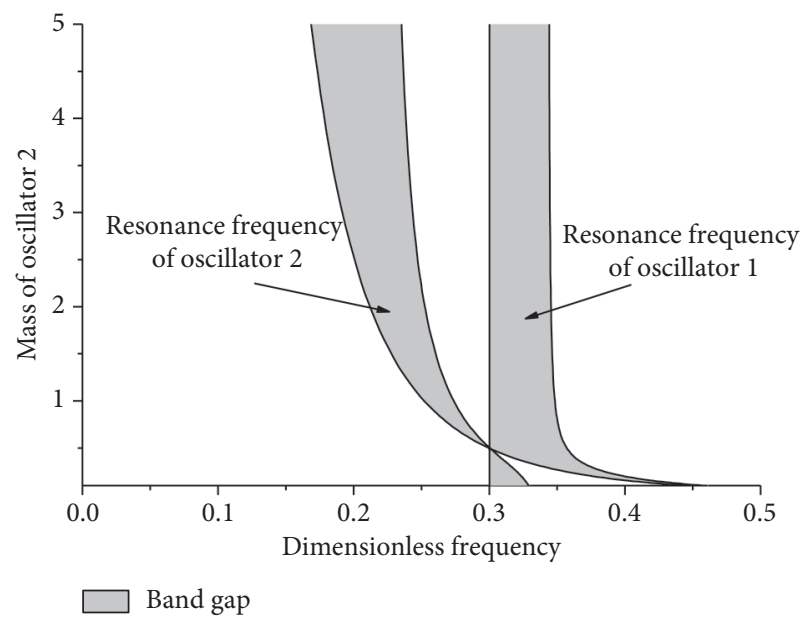

FIGURE 10: Mass effects of oscillator 2 on band gap of the LRPCs beam.

3.2.2. Stiffness of the Oscillator. Remaining the stiffness of oscillator 2 constant and varying the stiffness of oscillator 1 from 0 to 0.5 , the stiffness effects of oscillator 1 on the band gap of LRPCs beam can be analyzed. The band gap property of LRPCs beam is shown in Figure 11.

As can be seen from Figure 11, when the stiffness of oscillator 1 varies, the band gap of the LRPCs beam with two oscillators also has three stages: double-band gaps stage, coupled single-band gap stage, and separated-band gaps stage. During the process, the stiffness of the oscillator varies, and its resonance frequency also varies. In the first stage, there are two independent band gaps, in which the lowfrequency one is generated by the resonance of oscillator 1 , and the high-frequency one is generated by the resonance of oscillator 2. When the stiffness of oscillator 1 increases, the band gap gradually moves towards high frequency, and the band gap width first increases and then decreases; namely, there is the widest band gap (enlarged window in Figure 11). In the first stage, stiffness of the oscillator is small initially, the coupling resonance between the oscillator and the beam is extremely weak, and thus the local resonance band gap is not obvious and extremely narrow. As the stiffness of oscillator 1 increases, the coupling resonance characteristic is 


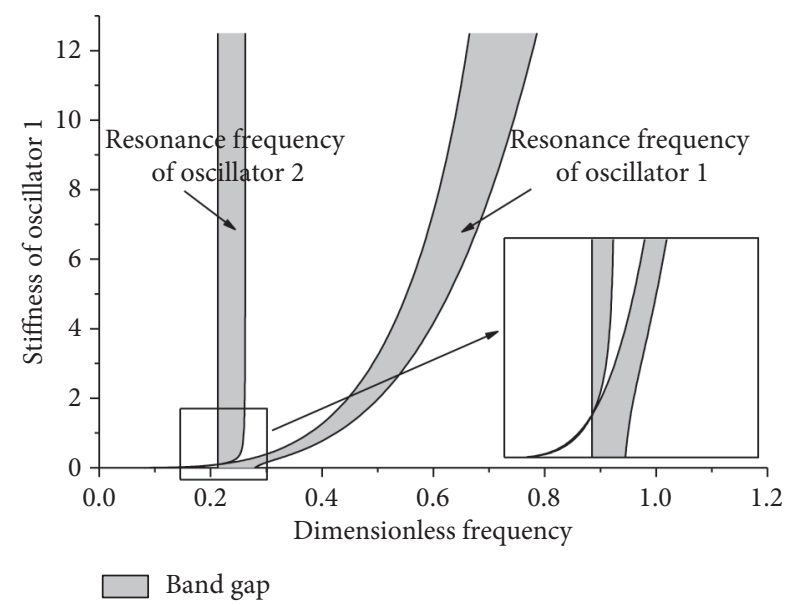

FIGURE 11: Stiffness effects of oscillator 1 on band gap of the LRPCs beam.

enhanced, and the band gap becomes wider. Besides, as the stiffness of oscillator increases, the resonance frequency increases, leading the band gap to move towards high frequency. When the band gap approaches the high-frequency band gap, the band gap narrows due to the compression of the high-frequency one, while the width of the high-frequency band gap is slightly increased because of the counteracting effects of the low-frequency one. In the second stage, the resonance frequency of oscillator 1 is equal to that of oscillator 2, and the two locally resonant band gaps are precisely encountered, and the double-band gaps couple into a single one. In the third stage, as the stiffness of oscillator 1 increases, the coupled single band gap splits into two band gaps, in which the low-frequency band gap is generated by the resonance of oscillator 2 , and the high-frequency band gap is generated by the resonance of oscillator 1. During the process, the position of high-frequency band gap moves towards higher frequency, and the high-frequency band gap widens, and the attenuation effect is enhanced. In fact, when spring stiffness of the oscillator increases, the oscillator can be considered fixed to the beam, and thus the band gap tends to be caused by the periodically concentrated mass, which is consistent with the variation rule of locally resonant band gap of beam with single oscillator [24]. Meanwhile, the starting frequency of low-frequency band gap remains constant, and its width increases and tends to be constant as the high-frequency band gap further moves away. This is because the high-frequency band gap is gradually away from the low-frequency one, and the compression is gradually weakened, and thus, the low-frequency one becomes wider. When the high-frequency band gap is further away, the lowfrequency band gap will not be affected.

Similarly, keeping the stiffness of oscillator 1 constant and only varying the stiffness of oscillator 2, the stiffness effects of oscillator 2 on band gap of LRPCs beam can be analyzed. The band gap property of LRPCs beam is shown in Figure 12.

As can be seen from Figure 12, the stiffness of oscillator 2 shows similar effects on band gap of LRPCs beam to that of oscillator 1. The difference is that oscillator 2 has a larger

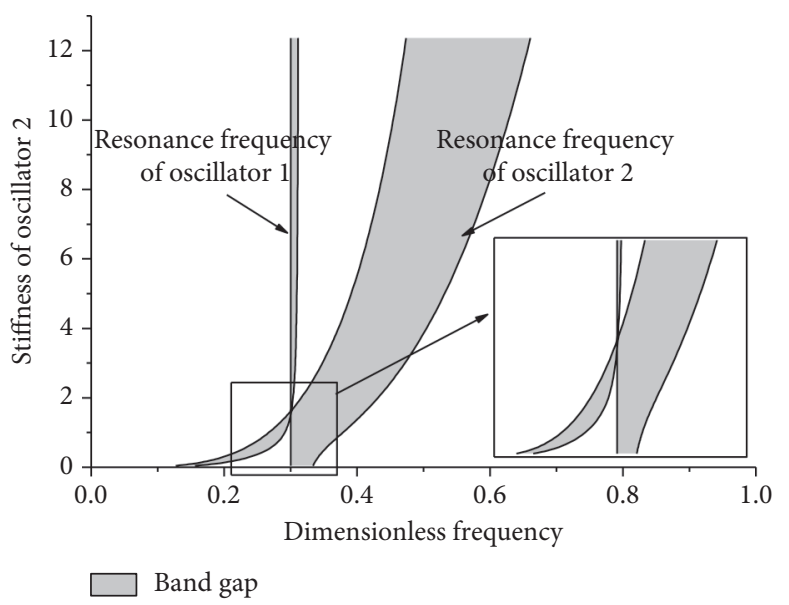

FIGURE 12: Stiffness effects of resonator 2 on band gap of the LRPCs beam.

mass and thus a stronger resonance intensity, so the lowfrequency band gap is relatively wider in the first stage (enlarged window in Figure 12). Besides, in the third stage, the low-frequency band gap is compressed and has an extremely small width because of its strong resonance intensity.

3.2.3. Mass (Stiffness) of the Oscillator at Its Fixed Resonance Frequency. By the resonance frequency of oscillators fixed, the effects of oscillator mass (or stiffness) on the band gap of LRPCs beam can be obtained. The band gap property of LRPCs beam is shown in Figures 13 and 14 .

As can be seen from Figure 13, the starting frequencies of the band gaps both remain constant because the resonance frequencies of the oscillators are fixed. When the mass of oscillator 1 is increased, the resonance intensity of oscillator 1 is enhanced, making the width of the high-frequency band gap increase, while the compression effect on the low-frequency band gap is enhanced, resulting in the width decrease of the low-frequency one.

As shown in Figure 14, when the mass of oscillator 2 increases, the resonance intensity of oscillator 2 increases, making the widths of both the low-frequency band gap and the high-frequency one increase. It indicates that when the resonance frequencies are fixed, the band gaps can be widened simultaneously by increasing the mass of the lowfrequency one.

3.3. Effects of External Load. The beams are usually subjected to external loads in practice. For example, in civil engineering, the existence of beam resting on the foundation is often encountered [35]. Usually, the foundation can be assumed as the Winkler foundation, which provides a uniform spring force for the beam. The spring force can be expressed as $f=c y$, where $c$ is the stiffness of foundation, and $y$ is the displacement of the beam. To investigate the effects of uniform spring force on the band gap, the stiffness of Winkler foundation can be defined as ratio $c=\gamma c_{0}$, where 


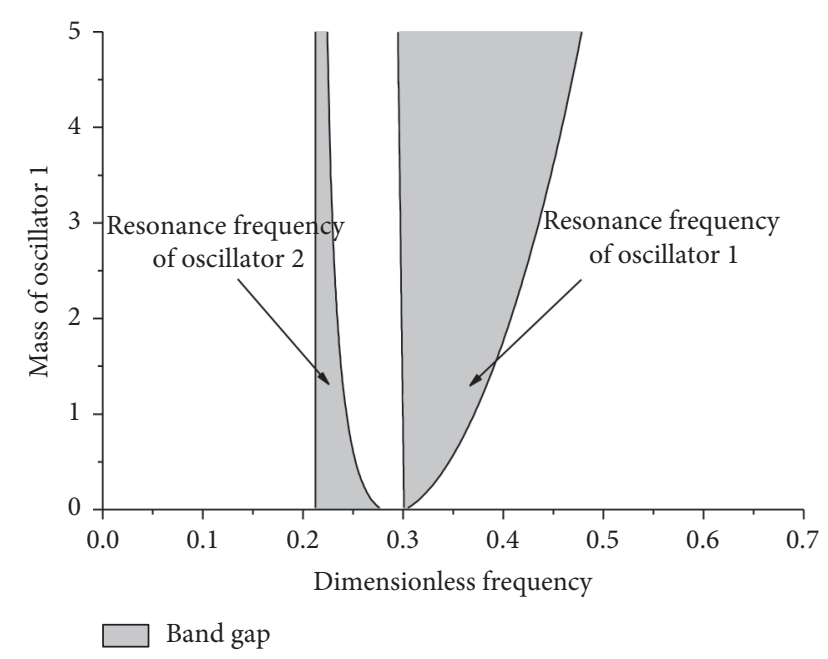

FIGURE 13: Mass effects of oscillator 1 on band gap of the LRPCs beam.

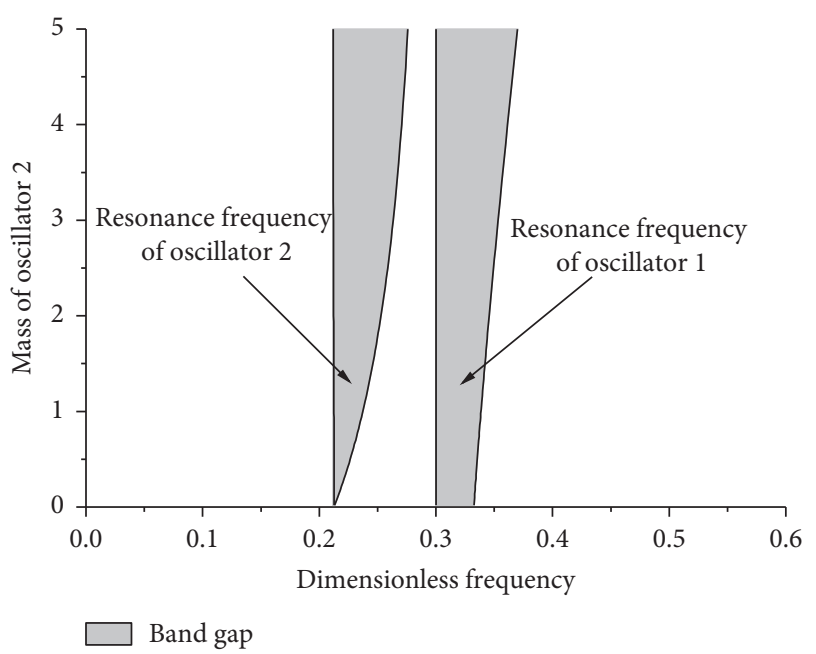

FIGURE 14: Mass effects of oscillator 2 on band gap of the LRPCs beam.

$c_{0}=10^{6} \mathrm{~N} / \mathrm{m}^{3}$. In this case, the governing equation of (1) can be transformed into

$$
E(x) I(x) \frac{\partial^{4} y(x, t)}{\partial x^{4}}+\rho(x) A(x) \frac{\partial^{2} y(x, t)}{\partial t^{2}}+b c y(x, t)=0,
$$

where $b$ is the width of the beam.

Analogically, taking the LRPCs beam model with 2 oscillators ( $m=2$, the same as Part 2.3) as an example, the effects of the uniform spring force on the LRPCs beam are shown in Figure 15.

As shown in Figure 15, there are three band gaps in the LRPCs beam, in which the lowest one is caused by the spring force, and the others are the locally resonant ones caused by the local resonance of the oscillators. When the foundation stiffness increases, the spring force increases, and all the three band gaps show an initial rapid increase but then

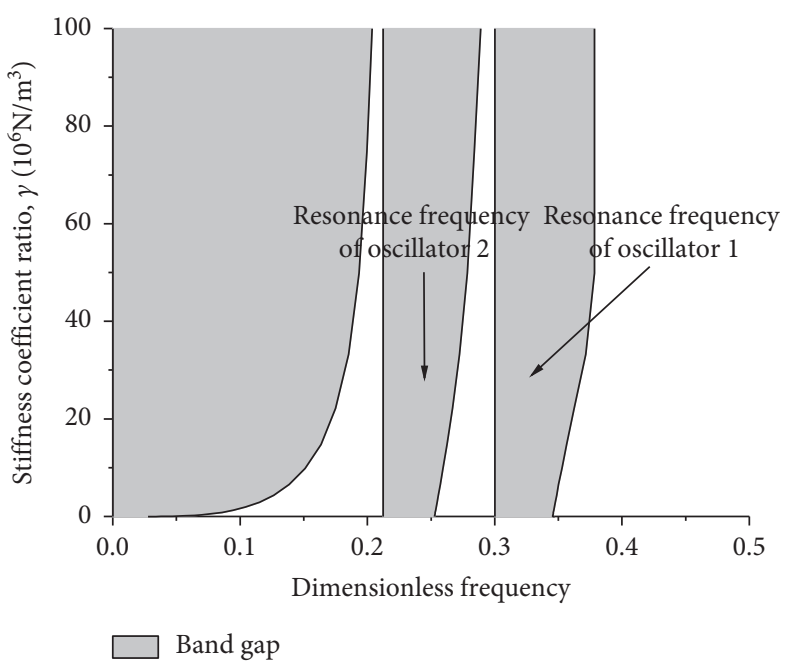

FIGURE 15: Effects of uniform spring force on band gaps.

increase constantly. Besides, the starting frequencies of all the band gaps keep constant. In fact, the starting frequency of LR band gap is closely related to the local resonance of the oscillator, and the increase of foundation stiffness only enhances the external spring load. Thus, the starting frequencies of LR band gaps keep constant. Generally, the variation range of foundation stiffness above is valid in practice, such as the soft soil foundation with low stiffness and the frozen soil foundation with high stiffness (up to $200 \times 10^{6} \mathrm{~N} / \mathrm{m}^{3}$ ). Then, knowing the effects of external spring force on the band gap is conducive to the vibration attenuation design of the beam-foundation system.

In can be concluded that the oscillator parameters and external load can affect both band gap location and band gap width. Therefore, to fabricate an optimized LRPCs structure, which is of the goal-oriented band gaps according to the need of vibration reduction, it is better to design the structure by varying these parameters based on above influence law.

\section{Vibration Attention of LRPCs Rubber Concrete Beam}

Generally, various kinds of vibration exist in our surroundings, for example, the ground vibration induced by railway, a kind of nuisance vibration related closely to our daily life. In civil engineering, concrete beam is often used as the foundation beam under building. Therefore, the theory of LRPCs can be introduced into the foundation beam to attenuate the ground vibration. Based on the above parameter analysis, a configuration of rubber concrete is presented to form LRPCs beam, and the LRPCs rubber concrete beam is shown in Figure 16. As shown in Figure 16, the rubber forms the cylindrical shell in concrete. As is wellknown, rubber shell has a low modulus $\left(\times 10^{5} \mathrm{~Pa}\right)$, and it is so soft. The rubber shell can be regarded as the soft coating layer, and it acts as the spring for oscillators in LRPCs rubber concrete beam. Meanwhile, the hard concrete core inside the rubber shell can act as the mass in LRPCs concrete beam, 


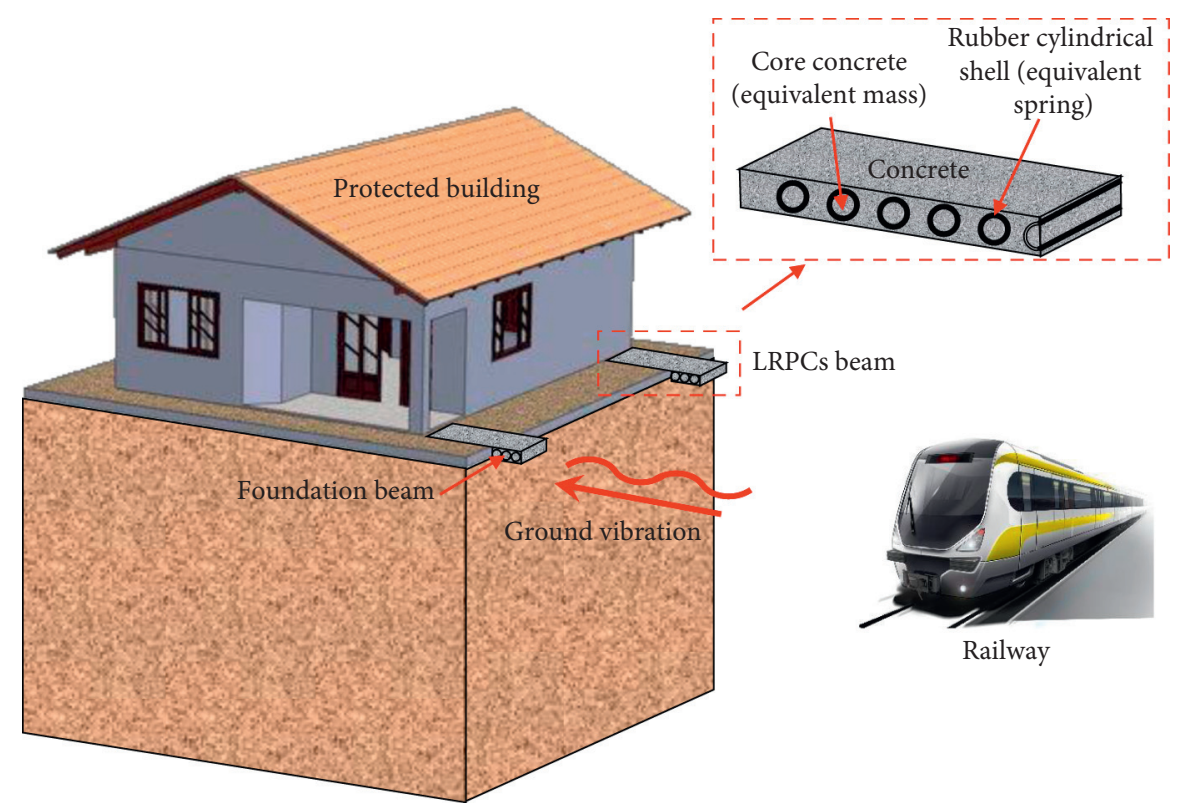

Figure 16: Vibration waves induced by railway and the LRPCs rubber concrete foundation beam.

namely, the soft rubber shell and the hard concrete core form the local resonator together. In this case, the LRPCs rubber concrete beam can be designed to attenuate the ground vibration induced by the railway. The design flow chart of LRPCs rubber beam is shown in Figure 17.

As shown in Figure 17, the main frequencies of isolated vibration must be given firstly. The isolated vibration is induced by the railway, and Figure 18 shows the acceleration record of railway $[45,46]$. As shown, the main frequencies of railway excitation range from 50 to $60 \mathrm{~Hz}$ and 115 to $125 \mathrm{~Hz}$. Secondly, considering that the rubber concrete beam is laying on the ground (elastic foundation), the beam suffers the reaction of foundation (Winkler foundation). The concrete beam is defined as follows: cross-section $b \times h=0.2 \mathrm{~m} \times 0.07 \mathrm{~m}$, Young's modulus $E_{c}=3.45 \times 10^{10} \mathrm{~Pa}$, density $\rho_{c}=2450 \mathrm{~kg} / \mathrm{m}^{3}$, Poisson's ratio $v_{c}=0.3$. And assume that the stiffness of Winkler foundation is defined as $c=30 \times 10^{6} \mathrm{~N} / \mathrm{m}^{3}$. Thirdly, choose appropriate oscillators. For convenience, we only choose one type of oscillator. Fourthly, determine the parameters of the local resonators. The mass and the spring equivalent stiffness can be adjusted by the radius and thickness of rubber shell, namely, the filling rate of ground rubber. The higher the filling rate of the rubber is, the lower the equivalent stiffness of the spring would be. In this paper, the parameters of rubber shell are as follows: Young's modulus $E_{r}=1.175 \times 10^{5} \mathrm{~Pa}$, density $\rho_{r}=1300 \mathrm{~kg} / \mathrm{m}^{3}$, Poisson's ratio $\nu_{\mathrm{r}}=0.42$, inner radius $r_{r}=0.0255 \mathrm{~m}$, thickness of rubber shell $t_{r}=0.01 \mathrm{~m}$, equivalent stiffness coefficient of rubber shell $k=5.216 \times 10^{5} \mathrm{~N} / \mathrm{m}$; mass of concrete core $m=1.94 \mathrm{~kg}$; oscillators displaced on beam at equal interval $a=0.35 \mathrm{~m}$. Then, the equivalent inertia moment (deducting rubber shell) of the concrete beam is $\mathrm{I}=2.5682 \times 10^{-6} \mathrm{~m}^{4}$. Figure 19 shows the band structure of LRPCs rubber concrete beam, and it can be seen that two band gaps appear in the beam $(<62 \mathrm{~Hz}$ and $114-123 \mathrm{~Hz})$. The main frequencies of railway excitation are just located within

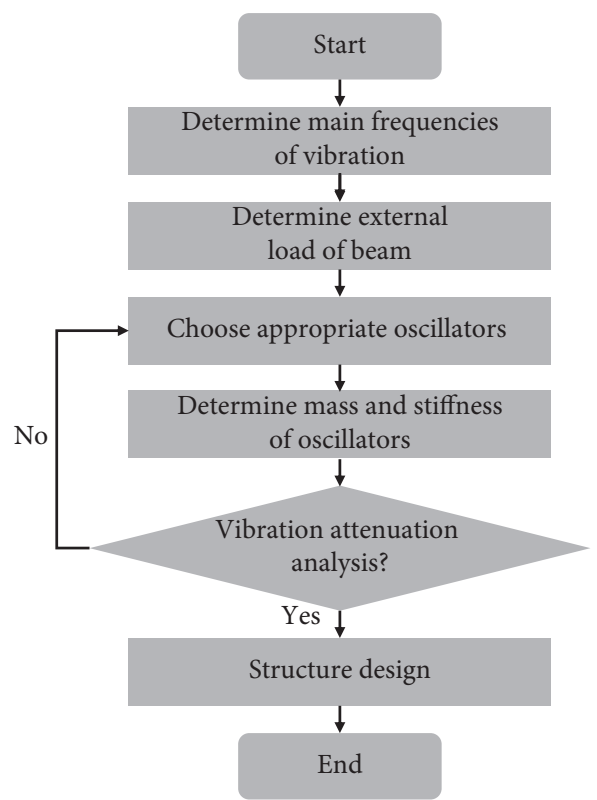

Figure 17: Design flow chart of the LRPCs beam.

the band gaps of the designed structure. Furthermore, the transient response of the LRPCs rubber concrete beam is conducted by FEM to analyze its vibration attenuation efficiency. In this paper, at least 6-cell structure is used, and the FEM model and transient acceleration response of the LRPCs rubber concrete beam are shown in Figures 20 and 21 , respectively. It can be seen that the LRPCs rubber concrete beam can attenuate the railway vibration waves effectively. Finally, based on the above theoretical calculation, the designed LRPCs rubber concrete beam is determined. Following the above design flow chart, the research can provide a reference for the vibration isolation of LRPCs beams in civil engineering fields. 


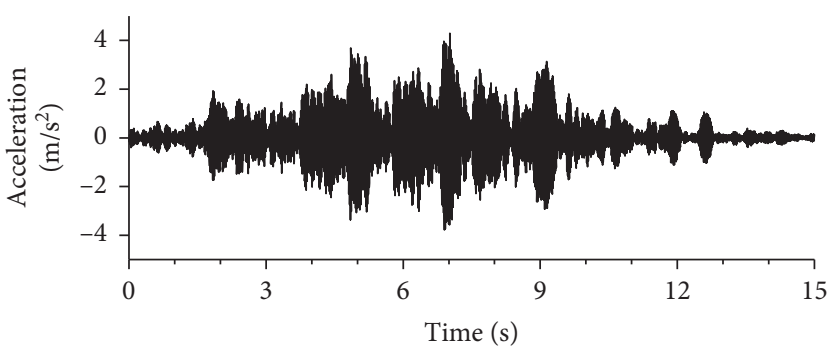

(a)

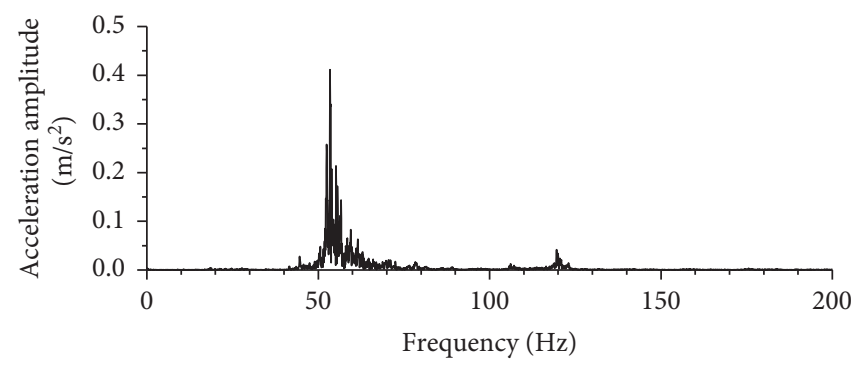

(b)

FIgURE 18: Acceleration record of railway.

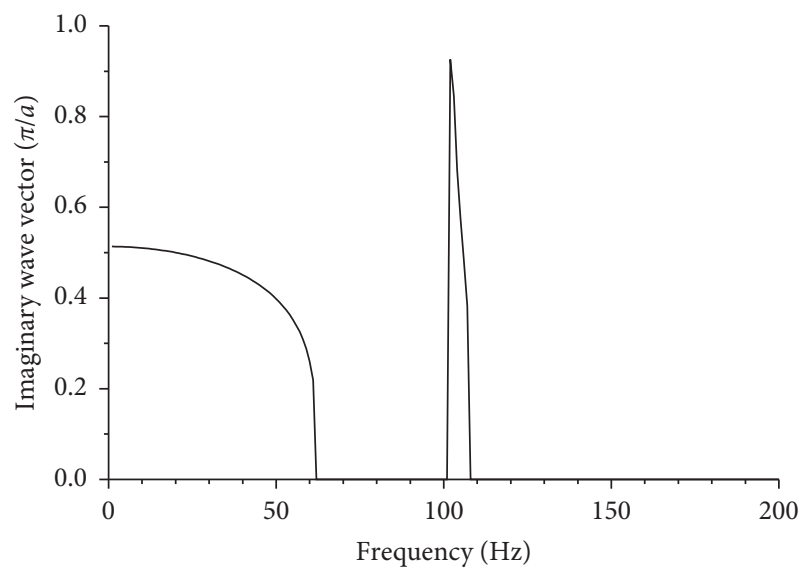

FIGURE 19: Band gaps of the LRPCs rubber concrete beam laying on foundation.

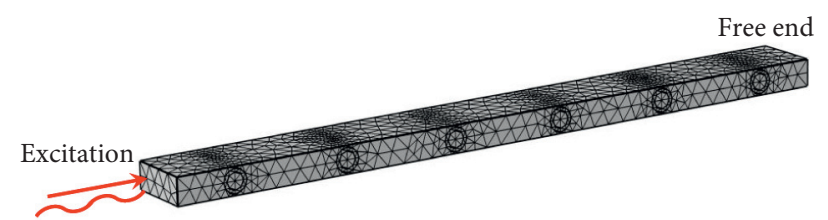

FIGURE 20: FEM model of the LRPCs rubber concrete beam (6-cell).

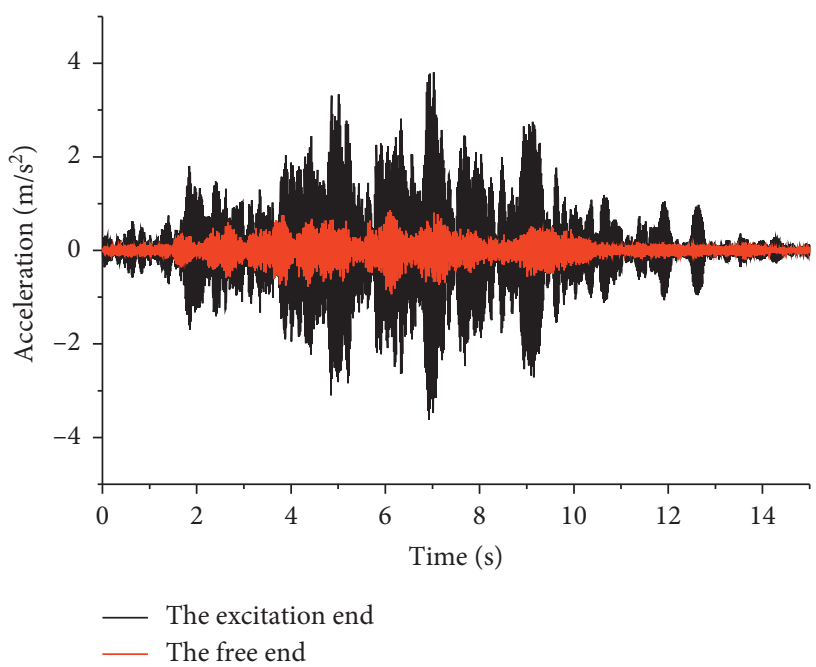

FIGURE 21: Acceleration time domain analysis of the LRPCs rubber concrete beam.

\section{Conclusions}

The multifrequency LRPCs beam with distributed multioscillators is proposed, and the band gap is figured out based on transfer matrix method (TMM) and Bloch's theorem. Besides, the parameter effects on the band gaps are investigated. Finally, a LRPCs rubber concrete beam is designed to show its application in isolating the railway vibration. Main conclusions can be drawn as follows:

(1) The distributed LRPCs beam has multifrequency band gaps, and the number of the gaps is equal to that of the local oscillators. Compared with other types of LRPCs beam, the distributed one can reduce the stress concentration when subjected to vibration.

(2) The oscillator interval has no effects on the band gaps, making it more convenient to design structures.

(3) Individual changes of oscillator mass or stiffness affect the band gap location and width. When the resonance frequency of oscillator is fixed, the starting frequency of the gap keeps constant. And increasing the oscillator mass in high-frequency part widens the high-frequency band gap, while increasing the oscillator mass in low-frequency part widens both high-frequency and low-frequency band gaps.

(4) The external loads, such as the uniform spring force encountered in civil engineering, are conducive to the generation of band gaps, and when the spring force increases, all the band gaps widen with the starting frequencies keeping constant.

(5) Taken together, a LRPCs rubber beam is designed, and it shows the good vibration isolation of LRPCs beam in civil engineering. By the design flow chart, the research can provide a reference for vibration isolation of LRPCs beams in civil engineering fields.

Finally, it should be noted that the external load in this paper is mainly concerned with the common spring force provided by the Winkler foundation. Moreover, the external loads contain other kinds of force, like the prestressing force provided by the steel in the concrete. However, this would be another systematic study with exhaustive computations, and thus, we leave it as an open problem. 


\section{Data Availability}

The data used to support the findings of this study are available from the corresponding author upon request.

\section{Conflicts of Interest}

The authors declare that there are no conflicts of interest regarding the publication of this paper.

\section{Acknowledgments}

This study was supported by the National Natural Science Foundation (Grant No. 51108252) and Shandong Transportation Science and Technology Project (Grant No. 2017B59).

\section{References}

[1] M. S. Kushwaha, P. Halevi, and L. Dobrzynski, "Acoustic band structure of periodic elastic composites," Physical Review Letters, vol. 1993, no. 71, pp. 2022-2025, 1993.

[2] M. S. Kushwaha and P. Halevi, "Band-gap engineering in periodic elastic composites," Applied Physics Letters, vol. 64, no. 9, pp. 1085-1087, 1994.

[3] M. S. Kushwaha, P. Halevi, G. Martínez, L. Dobrzynski, and B. Djafari-Rouhani, "Theory of acoustic band structure of periodic elastic composites," Physical Review B, vol. 49, no. 4, pp. 2313-2322, 1994.

[4] M. Sigalas and E. N. Economou, "Band structure of elastic waves in two dimensional systems," Solid State Communications, vol. 86, no. 3, pp. 141-143, 1993.

[5] J. O. Vasseur, B. Djafari-Rouhani, L. Dobrzynski, M. S. Kushwaha, and P. Halevi, "Complete acoustic band gaps in periodic fibre reinforced composite materials: the carbon/ epoxy composite and some metallic systems," Journal of Physics: Condensed Matter, vol. 6, no. 42, pp. 8759-8770, 1994.

[6] M. M. Sigalas and E. N. Economou, "Attenuation of multiplescattered sound," Europhysics Letters (EPL), vol. 36, no. 4, pp. 241-246, 1996.

[7] J. V. Sánchez-Pérez, D. Caballero, R. Mártinez-Sala et al., "Sound attenuation by a two-dimensional array of rigid cylinders," Physical Review Letters, vol. 80, no. 24, pp. 5325-5328, 1998.

[8] D. J. Mead, "A new method of analyzing wave propagation in periodic structures; Applications to periodic timoshenko beams and stiffened plates," Journal of Sound and Vibration, vol. 104, no. 1, pp. 9-27, 1986.

[9] D. J. Mead, "Free wave propagation in periodically supported, infinite beams," Journal of Sound and Vibration, vol. 11, no. 2, pp. 181-197, 1970.

[10] G. Wang, L. H. Shao, Y. Z. Liu et al., "Accurate evaluation of lowest band gaps in ternary locally resonant phononic crystals," Chinese Physics, vol. 15, no. 8, pp. 1843-1848, 2006.

[11] W. M. Kuang, Z. L. Hou, and Y. Y. Liu, "The effects of shapes and symmetries of scatterers on the phononic band gap in 2D phononic crystals," Physics Letters A, vol. 332, no. 5-6, pp. 481-490, 2004.

[12] M. M. Sigalas, "Elastic wave band gaps and defect states in two-dimensional composites," The Journal of the Acoustical Society of America, vol. 101, no. 3, pp. 1256-1261, 1997.

[13] M. Torres, F. R. Montero de Espinosa, and J. L. Aragón, "Ultrasonic wedges for elastic wave bending and splitting without requiring a full band gap," Physical Review Letters, vol. 86 , no. 19 , pp. 4282-4285, 2001.

[14] M. M. Sigalas and E. N. Economou, "Elastic and acoustic wave band structure," Journal of Sound and Vibration, vol. 158, no. 2, pp. 377-382, 1992.

[15] Z. Liu, X. J. Zhang, Y. W. Mao et al., "Locally resonant sonic materials," Science, vol. 289, no. 5485, pp. 1734-1736, 2000.

[16] Z. Liu, C. T. Chan, P. Sheng, A. L. Goertzen, and J. H. Page, "Elastic wave scattering by periodic structures of spherical objects: theory and experiment," Physical Review B, vol. 62, no. 4, pp. 2446-2457, 2000.

[17] C. Li, L. Miao, Q. You, H. Fang, X. Liang, and L. Lei, "Effects of material parameters on the band gaps of two-dimensional three-component phononic crystals," Applied Physics A, vol. 125, no. 3, p. 170, 2019.

[18] E. D. Nobrega, F. Gautier, A. Pelat, and J. M. C. Dos Santos, "Vibration band gaps for elastic metamaterial rods using wave finite element method," Mechanical Systems and Signal Processing, vol. 79, pp. 192-202, 2016.

[19] F.-L. Li, Y.-S. Wang, C. Zhang, and G.-L. Yu, "Boundary element method for band gap calculations of two-dimensional solid phononic crystals," Engineering Analysis With Boundary Elements, vol. 37, no. 2, pp. 225-235, 2013.

[20] Y. Guo, L. Li, and K.-C. Chuang, "Analysis of bending waves in phononic crystal beams with defects," Crystals, vol. 8, no. 1, p. 21, 2018.

[21] G. Wang, J. H. Wen, and X. S. Wen, "Locally resonant elastic wave band gaps in flexural vibrations of slender beams," Chinese Journal of Mechanical Engineering, vol. 41, no. 10, pp. 107-110, 2005.

[22] D. Yu, Y. Liu, G. Wang, H. Zhao, and J. Qiu, "Flexural vibration band gaps in Timoshenko beams with locally resonant structures," Journal of Applied Physics, vol. 100, no. 12, Article ID 124901, 2006.

[23] D. L. Yu, Y. Z. Liu, H. G. Zhao et al., "Flexural vibration band gaps in Euler-Bernoulli beams with locally resonant structures with two degrees of freedom," Physical Review B, vol. 73, no. 6, Article ID 064301, 2006.

[24] Y. Xiao, Locally Resonant Structures: Band Gap Tuning and Properties of Vibration and Noise Reduction, Graduate School of National University of Defense Technology, Changsha, China, 2012, in Chinese.

[25] J. Zhou, K. Wang, D. Xu, and H. Ouyang, "Multi-low-frequency flexural wave attenuation in Euler-Bernoulli beams using local resonators containing negative-stiffness mechanisms," Physics Letters A, vol. 381, no. 37, pp. 3141-3148, 2017.

[26] B. Sharma and C. T. Sun, "Local resonance and Bragg bandgaps in sandwich beams containing periodically inserted resonators," Journal of Sound And Vibration, vol. 364, pp. 133-146, 2016.

[27] R. Chen and T. Wu, "Vibration reduction in a periodic truss beam carrying locally resonant oscillators," Journal of Vibration and Control, vol. 22, no. 1, pp. 270-285, 2016.

[28] R. Chen and T. Wu, "Dynamic characteristics of a periodic rib-skin structure," Journal of Vibration and Control, vol. 22, no. 3, pp. 662-677, 2016.

[29] E. J. P. Miranda, E. D. Nobrega, A. H. R. Ferreira, and J. M. C. Dos Santos, "Flexural wave band gaps in a multiresonator elastic metamaterial plate using Kirchhoff-Love theory," Mechanical Systems and Signal Processing, vol. 116, pp. 480-504, 2019.

[30] Z. Tong, X. Qi, and L. Li, "A method for considering a distributed spring constant for studying the flexural vibration of an Euler-beam with lightweight multistage local resonators," 
Journal of Vibroengineering, vol. 20, no. 7, pp. 2563-2575, 2018.

[31] X. D. Wu, S. G. Zuo, T. X. Ni et al., "Study of the bandgap characteristics of a locally resonant phononic crystal beam with attached double oscillors in parallel," Journal of Vibration Engineering, vol. 2017, no. 1, pp. 79-85, 2017.

[32] Y. Xiao, J. Wen, and X. Wen, "Broadband locally resonant beams containing multiple periodic arrays of attached resonators," Physics Letters A, vol. 376, no. 16, pp. 1384-1390, 2012.

[33] Q. H. Wen Qi-Hua, S. G. Zuo Shu-Guang, and H. Wei Huan, "Locally resonant elastic wave band gaps in flexural vibration of multi-oscillators beam," Acta Physica Sinica, vol. 61, no. 3, Article ID 034301, 2012.

[34] S. G. Zuo, T. X. Ni, X. D. Wu et al., "Studies of band gaps in flexural vibrations of a locally resonant beam with novel multi-oscillator configuration," Journal of Vibration and Control, vol. 23, no. 10, pp. 1663-1674, 2017.

[35] M. Sato, S. Kanie, and T. Mikami, "Structural modeling of beams on elastic foundations with elasticity couplings," Mechanics Research Communications, vol. 34, no. 5-6, pp. 451-459, 2007.

[36] D. Yu, J. Wen, H. Shen, Y. Xiao, and X. Wen, "Propagation of flexural wave in periodic beam on elastic foundations," Physics Letters A, vol. 376, no. 4, pp. 626-630, 2012.

[37] Y. Zhang, Z. Q. Ni, L. H. Jiang et al., "Study of the bending vibration characteristic of phononic crystals beam-foundation structures by Timoshenko beam theory," International Journal of Modern Physics B, vol. 29, no. 20, Article ID 1550136, 2015.

[38] A. Hamdi, G. Abdelaziz, and K. Z. Farhan, "Scope of reusing waste shredded tires in concrete and cementitious composite materials: a review," Journal of Building Engineering, vol. 35, Article ID 102014, 2021.

[39] A. Siddika, M. A. A. Mamun, R. Alyousef, Y. H. M. Amran, F. Aslani, and H. Alabduljabbar, "Properties and utilizations of waste tire rubber in concrete: a review," Construction and Building Materials, vol. 224, pp. 711-731, 2019.

[40] R. Hassanli, O. Youssf, and J. E. Mills, "Experimental investigations of reinforced rubberized concrete structural members," Journal of Building Engineering, vol. 10, pp. 149$165,2017$.

[41] M. K. Ismail and A. A. A. Hassan, "Ductility and cracking behavior of reinforced self-consolidating rubberized concrete beams," Journal of Materials in Civil Engineering, vol. 29, no. 1, Article ID 04016174, 2017.

[42] K. Strukar, T. Kalman Šipoš, I. Miličević, and R. Bušić, "Potential use of rubber as aggregate in structural reinforced concrete element - a review," Engineering Structures, vol. 188, pp. 452-468, 2019.

[43] H. Li, Z. Han, and X. Zhu, "Seismic behavior of a frame-shear wall structure with crumb rubber concrete beam," Journal of Shenyang Jianzhu University (Nature Science), vol. 25, no. 2, pp. 149-155, 2013, in Chinese.

[44] Y. Xiao, J. Wen, D. Yu, and X. Wen, "Flexural wave propagation in beams with periodically attached vibration absorbers: band-gap behavior and band formation mechanisms," Journal of Sound And Vibration, vol. 332, no. 4, pp. 867-893, 2013.

[45] X. Pu and Z. Shi, "Surface-wave attenuation by periodic pile barriers in layered soils," Construction and Building Materials, vol. 180, no. 2018, pp. 177-187, 2018.
[46] M. Gao and Z. Shi, "A wave guided barrier to isolate antiplane elastic waves," Journal of Sound and Vibration, vol. 443, pp. 155-166, 2019. 